\title{
A single-nucleotide polymorphism in MMP9 is associated with decreased risk of steroid-induced osteonecrosis of the femoral head
}

\author{
Jieli Du1,2,*, Wanlin Liu', ${ }^{2,}$, Tianbo Jin ${ }^{3}$, Zhenqun Zhao ${ }^{2}$, Rui Bai ${ }^{2}$, Huiqin Xue ${ }^{2}$, Junyu \\ Chen $^{1,2}$, Mingqi Sun ${ }^{2}$, Xiyang Zhang ${ }^{3}$, Guoqiang Wang ${ }^{2}$, Jianzhong Wang ${ }^{2}$ \\ ${ }^{1}$ Inner Mongolia Medical University, Hohhot, Inner Mongolia, 010050, China \\ ${ }^{2}$ Department of Orthopedics and Traumatology, The 2nd affiliated Hospital of Inner Mongolia University, Hohhot, Inner \\ Mongolia, 010030, China \\ ${ }^{3}$ The College of Life Sciences, Northwest University, 710069, China \\ *Co-first authors, these authors contributed equally to this work
}

Correspondence to: Guoqiang Wang, email: wangguoqianggq@163.com

Jianzhong Wang, email: wangjianzhongwj@163.com

Keywords: MMP9, MMP2, single nucleotide polymorphisms, osteonecrosis of the femoral head, association study

Received: May 31, $2016 \quad$ Accepted: September 05, $2016 \quad$ Published: September 15, 2016

\section{ABSTRACT}

Osteonecrosis of the femoral head (ONFH) is a common hip joint disease, and steroid-induced ONFH accounts for a large number of cases. Here, we examined eight previously-identified single-nucleotide polymorphisms (SNPs) in the MPP2 and MPP9 genes of $\mathbf{2 8 5}$ steroid-induced ONFH patients and $\mathbf{5 0 7}$ healthy controls from northern China to determine whether these SNPs were associated with the risk of developing steroid-induced ONFH. Chi-squared tests and genetic model and haplotype analyses were used to evaluate associations. The rs2274755 SNP in MMP9 was associated with a decreased risk of steroid-induced ONFH in the allele, dominant, and additive models. Additionally, the "CGC" MMP9 haplotype was associated with a 0.69-fold decrease in the risk of steroid-induced ONFH. Although additional, larger population-based studies are needed to confirm these findings, our results reveal for the first time an association between a MMP9 SNP at the rs2274755 locus and a decreased risk of steroid-induced ONFH in a northern Chinese population.

\section{INTRODUCTION}

Osteonecrosis of the femoral head (ONFH) is a common hip joint disease that frequently causes disability. Nearly 20 million patients suffer from ONFH worldwide [1], with an estimated 8.12 million non-traumatic ONFH cases in 2013 in China alone [2]. Steroid-induced ONFH accounts for $24.1 \%$ of all ONFH cases [1]. Several studies demonstrate that only some patients develop ONFH within a few months of glucocorticoid (GC) therapys, suggesting that genetic factors may confer susceptibility or resistance to steroid-induced ONFH.

Matrix metalloproteinases (MMPs) are a family of zinc-dependent proteolytic enzymes that participate in morphogenesis, wound healing, tissue repair, and remodeling in response to injury, and glucocorticoids can inhibit the expression of MMPs. Garvican et al. [3] found that corticosteroids suppressed the expression of MMP-1,
MMP-3, MMP-13, gelatinase A (MMP-2), and gelatinase B (MMP-9) mRNA. Glucocorticoids also down-regulate MMP-2 expression by inducing microRNA 29c [4]. Dexamethasone might aid in the treatment of bacterial meningitis and acute lung injury by down-regulating MMP-9 expression [5, 6]. In addition, gelatinase A (MMP-2) and B (MMP-9) play crucial roles in bone remodeling [7]. Both gelatinase A (MMP-2) and gelatinase B (MMP-9) are produced by osteoblasts, and MMP-9 can also be synthesized by osteoclasts [8]. MMP-2 expression is upregulated in osteoarthritic cartilage [9], and MMP-9, which regulates growth plate angiogenesis and apoptosis of hypertrophic chondrocytes, may be essential for normal bone remodeling [10]. Both MMP-2 and MMP-9 expression are increased in the joints of rheumatoid arthritis patients $[11,12]$. Additionally, MMP-2 mRNA and protein levels are up-regulated in ONFH subjects, while MMP-9 protein is overproduced in osteoarthritic bone 
tissue [8]. Inactivating mutations in the $M M P 2$ gene result in bone and joint features of multicentric osteolysis with arthritis [13], and osteoclast MMP-9 expression increases in response to adrenocorticotropic hormone (ACTH) in murine models, confirming the effects of ACTH on early osteoclast differentiation [14]. Furthermore, observations that low ACTH levels are associated with microvascular osteonecrosis and that $\mathrm{ACTH}$ administration reduces this necrosis suggest that ACTH might be useful for decreasing the risk of osteonecrosis in humans [14]. Additionally, MMP-9 expression may serve as a marker of efficacy in the treatment of steroid-induced osteonecrosis using ACTH. MMP-2 and MMP-9 expression, which were both higher in steroid-induced ONFH patients than in controls, have only recently been investigated in Chinese populations.

Genetic polymorphisms are normal genetic variations found in more than $1 \%$ of the population that can influence protein transcription and the expression of related genes to contribute to an individual's disease susceptibility [15]. MMP2 and $M M P 9$ single nucleotide polymorphisms (SNPs) have been associated with several diseases. For example, rs1053605 and rs243849 polymorphisms are associated with stroke outcome [16], but not with obesity [17], thoracic aortic dissection [18], or endometriosis [19]. rs243847 polymorphism might increase the risk of non-vertebral osteoporotic fractures [20] and intracranial aneurysms in male patients [21]. rs243832 and rs7201 polymorphisms are associated with an increased risk of endometriosis [19]. rs3918249 polymorphism is associated with a decreased risk of glaucoma, especially in Caucasian patients [22], and an increased risk of childhood-onset asthma [23]; in the same study, rs2274755 polymorphism was also associated with an increased risk of asthma. Gao et al. [24] suggested that the rs3918254 locus may be susceptible to primary angle-closure glaucoma in Chinese patients. However, associations between these eight SNPs and steroidinduced ONFH have not yet been examined. The aim of this study was to determine whether s(SNPs in the MMP2 and $M M P 9$ genes were associated with the risk of steroidinduced $\mathrm{ONFH}$ in a northern Chinese patient population.

\section{RESULTS}

Sex and age distributions for ONFH patients and controls are shown in Table 1. 285 ONFH patients (112 female, 173 male) and 507 controls (111 female, 396 male) were recruited for the present study. The mean ages were $41.88 \pm 12.79$ years for patients and $47.43 \pm$ 9.74 years for controls.

Eight SNPs in $M M P 2$ and $M M P 9$ were analyzed in this study. Allele frequencies and basic information for all SNPs are shown in Table 2. All eight SNPs exhibited Hardy-Weinberg equilibrium in control subjects $(p>0.05)$, while rs2274755 was associated with a decreased risk of steroid-induced ONFH $(\mathrm{OR}=0.70,95 \%$ CI: $0.51-0.96, p=0.025)$.

We then examined whether harboring the minor allele for each SNP compared to the wild-type allele represented a risk factor in the genetic models shown in Table 3. rs2274755 polymorphism in the MMP9 gene conferred a protective effect against steroid-induced $\mathrm{ONFH}$ in the dominant model both before $(\mathrm{OR}=0.66$, 95\% CI: $0.47-0.94, p=0.019)$ and after adjustment for age and gender $(\mathrm{OR}=0.68,95 \% \mathrm{CI}: 0.47-0.98, p=0.034)$, and in the additive model both before $(\mathrm{OR}=0.70,95 \%$ CI: $0.51-0.96, p=0.022)$ and after adjustment for age and gender $(\mathrm{OR}=0.70,95 \% \mathrm{CI}: 0.51-0.98, p=0.033)$. However, in the over-dominant model, rs2274755 polymorphism was only associated with a decreased risk of steroid-induced ONFH before adjustment for age and gender $(\mathrm{OR}=0.67,95 \% \mathrm{CI}: 0.47-0.96, p=0.026)$.

Finally, haplotype analysis detected three blocks in the MMP2 and MMP9 genes (Figures 1 and 2). rs243849 and rs243847, rs243832 and rs7201, and rs3918249, rs2274755, and rs3918254 had very strong linkage disequilibria; compared to the "CGC" wild-type, the "CTC" haplotype was associated with a decreased risk of steroid-induced ONFH (OR $=0.69,95 \%$ CI: 0.48-0.98, $p=0.039$ ) (Table 4).

However, after Bonferroni correction was applied to our data, there were no statistically significant associations between $M M P 2$ and $M M P 9$ SNPs and the risk of steroidinduced ONFH.

\section{DISCUSSION}

Genetic studies have provided insight into numerous diseases, including osteonecrosis. In this study, eight SNPs in the $M M P 2$ and $M M P 9$ genes that have previously been investigated in other diseases were examined in 792 subjects (285 patients with steroid-induced ONFH and 507 healthy controls) to determine whether they were associated with the risk of steroid-induced ONFH in a northern Chinese population. Our results suggest that rs2274755 MMP9 genetic polymorphism was associated with a decreased risk of steroid-induced ONFH. In addition, the "CGC" $M M P 9$ haplotype was associated with a 0.69 -fold decrease in the risk of steroid-induced ONFH.

MMP-9, an extracellular matrix (ECM)-metabolizing enzyme, plays a crucial role in bone remodeling. Overexpression of MMP-9 by osteoclasts is critical for digesting type I collagen and aggrecan, which are found mainly in cartilage [11]. MMP-9 expression is stimulated by RANKL, which is essential for osteoclastogenesis [25-27]. Fujisaki et al. [28] reported that RANKL induced MMP-9 production in osteoclasts and increased bone resorption in the presence of IL- $1 \alpha$. In addition, Guo et al. [29] showed that decreased membranetype matrixmetalloproteinases-1 (MT1-MMP) expression is involved in RANKL signaling in osteoblasts and 
Table 1: Characteristics of cases and controls in this study

\begin{tabular}{lccc}
\hline Variable(s) & $\begin{array}{c}\text { Case } \\
(\boldsymbol{n}=\mathbf{2 8 5})\end{array}$ & $\begin{array}{c}\text { Control } \\
(\boldsymbol{n}=\mathbf{3 0 8})\end{array}$ & $\boldsymbol{p}$ value \\
\hline Sex N(\%) & & & $<0.001^{\mathrm{a}}$ \\
Male & $173(60.7)$ & $396(78.1)$ & \\
Female & $112(39.3)$ & $111(21.9)$ & $<0.001^{\mathrm{b}}$ \\
Age, year $($ mean $\pm \mathrm{SD})$ & $41.88 \pm 12.79$ & $47.43 \pm 9.74$ & \\
\hline
\end{tabular}

${ }^{a}$ Two-sided Chi-squared test.

${ }^{b}$ Independent samples $t$ test.

Table 2: Allele frequencies in cases and controls and odds ratio estimates for steroid-induced ONFH

\begin{tabular}{|c|c|c|c|c|c|c|c|c|c|c|}
\hline \multirow{3}{*}{$\begin{array}{c}\text { SNP ID } \\
\text { rs1053605 }\end{array}$} & \multirow{3}{*}{$\begin{array}{c}\text { Gene } \\
\text { MMP2 }\end{array}$} & \multirow{3}{*}{$\begin{array}{c}\text { Position } \\
16 \mathrm{q} 12.2\end{array}$} & \multirow{3}{*}{$\begin{array}{c}\begin{array}{c}\text { Alleles } \\
\text { A/B }\end{array} \\
\mathrm{T} / \mathrm{C}\end{array}$} & \multicolumn{2}{|c|}{ MAF } & \multirow{3}{*}{$\begin{array}{c}\begin{array}{c}\boldsymbol{p}^{\mathrm{a}} \text { value } \\
\text { for HWE }\end{array} \\
0.843\end{array}$} & \multirow{3}{*}{$\begin{array}{c}\text { ORs } \\
0.75\end{array}$} & \multirow{2}{*}{\multicolumn{2}{|c|}{$95 \% \mathrm{CI}$}} & \multirow{3}{*}{$\begin{array}{c}\boldsymbol{p}^{\mathbf{b}} \\
0.085\end{array}$} \\
\hline & & & & \multirow{2}{*}{$\frac{\text { case }}{0.11}$} & \multirow{2}{*}{$\begin{array}{c}\text { control } \\
0.15\end{array}$} & & & & & \\
\hline & & & & & & & & 0.54 & 1.04 & \\
\hline rs243849 & MMP2 & $16 \mathrm{q} 12.2$ & $\mathrm{~T} / \mathrm{C}$ & 0.21 & 0.20 & 1.000 & 1.03 & 0.78 & 1.35 & 0.858 \\
\hline rs243847 & MMP2 & $16 q 12.2$ & $\mathrm{C} / \mathrm{T}$ & 0.82 & 0.68 & 0.117 & 1.20 & 0.97 & 1.47 & 0.090 \\
\hline rs 243832 & MMP2 & $16 q 12.2$ & $\mathrm{C} / \mathrm{G}$ & 0.57 & 0.62 & 0.347 & 0.92 & 0.74 & 1.13 & 0.424 \\
\hline rs7201 & MMP2 & $16 \mathrm{q} 12.2$ & $\mathrm{C} / \mathrm{A}$ & 0.37 & 0.34 & 0.349 & 1.07 & 0.85 & 1.36 & 0.551 \\
\hline rs3918249 & MMP9 & $20 \mathrm{q} 13.12$ & $\mathrm{~T} / \mathrm{C}$ & 0.44 & 0.48 & 0.613 & 0.93 & 0.75 & 1.16 & 0.525 \\
\hline rs2274755 & MMP9 & $20 \mathrm{q} 13.12$ & $\mathrm{~T} / \mathrm{G}$ & 0.12 & 0.18 & 0.729 & 0.70 & 0.51 & 0.96 & $0.025^{*}$ \\
\hline rs3918254 & MMP9 & $20 \mathrm{q} 13.12$ & $\mathrm{~T} / \mathrm{C}$ & 0.28 & 0.23 & 0.664 & 1.19 & 0.93 & 1.54 & 0.173 \\
\hline
\end{tabular}

SNP single nucleotide polymorphism, HWE Hardy-Weinberg equilibrium, OR odds ratio, $95 \%$ CI 95\% confidence interval, MAF minor allele frequency.

$* p \leq 0.05$.

Bonferroni's multiple adjustment was applied to the level of significance, which was set at $p \leq 0.00104(0.05 / 48)$.

${ }^{\mathrm{a}} p$ was calculated by exact test.

${ }^{\mathrm{b}} p$ was calculated by Pearson Chi-squared test.

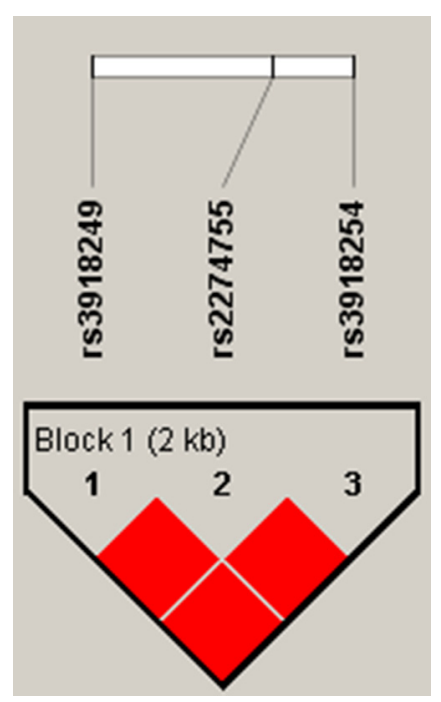

Figure 1: Linkage disequilibrium (LD) plots containing three SNPs from MMP9. Red squares display statistically significant associations between a pair of SNPs, as measured by D'; darker shades of red indicate higher D' 
Table 3: Genotypic model analysis of relationship between SNPs and steroid-induced ONFH risk

\begin{tabular}{|c|c|c|c|c|c|c|c|}
\hline \multirow{2}{*}{ Model } & \multirow{2}{*}{ Genotype } & \multirow{2}{*}{ Group $=$ control } & \multirow{2}{*}{ Group $=$ Hormone } & \multicolumn{2}{|c|}{ Without Adjustment } & \multicolumn{2}{|c|}{ With Adjustment } \\
\hline & & & & OR $(95 \%$ CI $)$ & $p^{\text {a }}$-value & OR (95\% CI) & $p^{\text {a }}$-value \\
\hline \multirow{3}{*}{ Codominant } & $\mathrm{G} / \mathrm{G}$ & $364(71.8 \%)$ & $226(79.3 \%)$ & 1 & \multirow{3}{*}{0.063} & 1 & \multirow{3}{*}{0.100} \\
\hline & $\mathrm{G} / \mathrm{T}$ & $133(26.2 \%)$ & $55(19.3 \%)$ & $0.67(0.47-0.95)$ & & $0.69(0.48-1.00)$ & \\
\hline & $\mathrm{T} / \mathrm{T}$ & $10(2 \%)$ & $4(1.4 \%)$ & $0.64(0.20-2.08)$ & & $0.56(0.17-1.92)$ & \\
\hline \multirow{2}{*}{ Dominant } & $\mathrm{G} / \mathrm{G}$ & $364(71.8 \%)$ & $226(79.3 \%)$ & 1 & \multirow{2}{*}{$0.019 *$} & 1 & \multirow{2}{*}{$0.034 *$} \\
\hline & $\mathrm{G} / \mathrm{T}-\mathrm{T} / \mathrm{T}$ & $143(28.2 \%)$ & $59(20.7 \%)$ & $0.66(0.47-0.94)$ & & $0.68(0.47-0.98)$ & \\
\hline \multirow{2}{*}{ Recessive } & G/G-G/T & $497(98 \%)$ & $281(98.6 \%)$ & 1 & \multirow{2}{*}{0.550} & 1 & \multirow{2}{*}{0.420} \\
\hline & $\mathrm{T} / \mathrm{T}$ & $10(2 \%)$ & $4(1.4 \%)$ & $0.71(0.22-2.28)$ & & $0.61(0.18-2.08)$ & \\
\hline \multirow{2}{*}{ Overdominant } & G/G-T/T & $374(73.8 \%)$ & $230(80.7 \%)$ & 1 & \multirow{2}{*}{$0.026^{*}$} & 1 & \multirow{2}{*}{0.055} \\
\hline & $\mathrm{G} / \mathrm{T}$ & $133(26.2 \%)$ & $55(19.3 \%)$ & $0.67(0.47-0.96)$ & & $0.70(0.48-1.01)$ & \\
\hline Log-additive & - & - & - & $0.70(0.51-0.96)$ & $0.022 *$ & $0.70(0.51-0.98)$ & $0.033^{*}$ \\
\hline
\end{tabular}

$* p \leq 0.05$.

Bonferroni's multiple adjustment was applied to the level of significance, which was set at $p \leq 0.00104(0.05 / 48)$.

$p$ values were calculated by Wald test by unconditional logistic regression adjusted for age and gender.

Table 4: The haplotype frequencies of $M M P 9$ polymorphisms and their association with steroidinduced ONFH risk

\begin{tabular}{ccccccccc}
\hline & & Haplotype & & & \multicolumn{2}{c|}{ Without Adjustment } & \multicolumn{2}{c}{ With Adjustment } \\
\hline & $\mathbf{r s 3 9 1 8 2 4 9}$ & rs2274755 & rs3918254 & Freq & OR (95\% CI) & $\boldsymbol{p}^{\mathbf{a}}$ & OR (95\% CI) & $\boldsymbol{p}^{\mathbf{b}}$ \\
\hline 1 & $\mathrm{C}$ & $\mathrm{G}$ & $\mathrm{C}$ & 0.3491 & 1 & - & 1 & - \\
2 & $\mathrm{~T}$ & $\mathrm{G}$ & $\mathrm{C}$ & 0.3169 & $0.88(0.68-1.13)$ & 0.310 & $0.91(0.70-1.18)$ & 0.470 \\
3 & $\mathrm{C}$ & $\mathrm{G}$ & $\mathrm{T}$ & 0.1976 & $1.06(0.80-1.42)$ & 0.680 & $1.03(0.76-1.40)$ & 0.850 \\
4 & $\mathrm{C}$ & $\mathrm{T}$ & $\mathrm{C}$ & 0.1364 & $0.67(0.48-0.95)$ & $0.026^{*}$ & $0.69(0.48-0.98)$ & $0.039 *$ \\
\hline
\end{tabular}

$* p \leq 0.05$ indicates statistical significance.

${ }^{a} p$ value were calculated by unconditional logistic regression.

${ }^{\mathrm{b}} p$ values were calculated by unconditional logistic regression adjusted for age and gender.

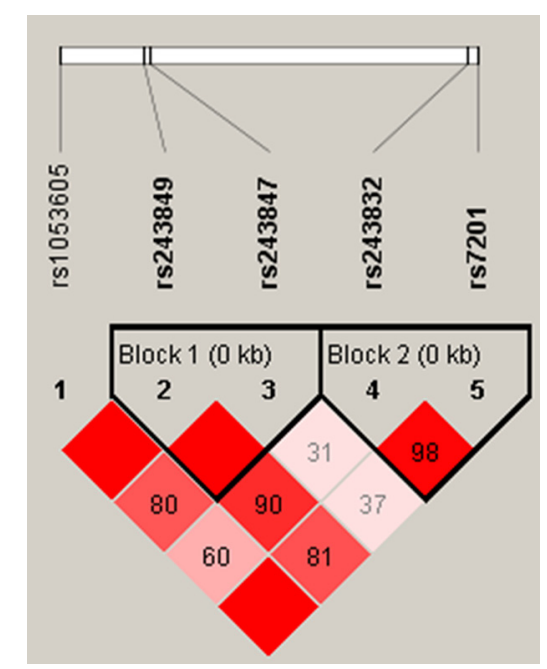

Figure 2: Linkage disequilibrium (LD) plots containing five SNPs from $M M P 2$. Red squares display statistically significant associations between a pair of SNPs, as measured by D'; darker shades of red indicate higher D'. 
the activation of osteoclasts. Thus, osteoclast-derived MMP-9 may contribute to bone resorption in steroidinduced ONFH. MMP-9 also facilitates the recruitment of inflammatory cells in the airways during allergic inflammation and chronic obstructive pulmonary disease [30, 31]. Therefore, MMP9 might also contribute to steroid-induced ONFH by affecting bone resorption or inflammatory responses. Additional studies are needed to characterize the exact mechanisms by which MMP-9 regulates steroid-induced ONFH.

The rs2274755 locus is located in an intron (boundary) of the MMP9 gene, and rs2274755 variations have been investigated in many studies. In a study of a Chinese Han population, no association was found between rs2274755 and susceptibility to either polypoidal choroidal vasculopathy or age-related macular degeneration [32]. Jimenez-Morales et al. found that rs2274755 polymorphism was associated with asthma in Mexican pediatric patients [23], as was the case in Nakashima et al.'s study in a Japanese population [33]. Furthermore, a functional study suggested that MMP-9 polymorphisms were associated with increased asthma pathogenesis [33]. Here, we found that rs2274755 polymorphism was associated with a decreased risk of steroid-induced ONFH in a northern Chinese population. Thus, rs2274755 variation may have a complex effect on inflammatory cells and capillaries in the femoral head, which may promote steroid-induced ONFH.

To our knowledge, the present study provides the first evidence that variation at the rs2274755 locus in the MMP9 gene is associated with a decreased risk of steroid-induced ONFH risk in the northern Chinese population. However, some limitations of this study should be considered when interpreting these results. First, the sample sizes were relative small, and additional studies should confirm these results in more patients. Second, all samples were collected at the Zhengzhou Traditional Chinese Medicine Traumatology Hospital in northern China, which may increase the type I error (false positive) rate for association studies. Additionally, the Bonferroni correction for multiple tests is important to account for false discovery rate. After correction, we found no statistically significant associations between $M M P 2$ and $M M P 9$ SNPs and the risk of steroid-induced ONFH. This may be due to our small sample size and strict SNP filtering criteria; furthermore, the Bonferroni correction is very conservative when applied to multiple tests. Continued sample collection will help to confirm our results in future studies.

Taken together, our results identified for the first time an association between rs2274755 MMP9 polymorphism and a decreased risk of steroid-induced ONFH in a northern Chinese population. Additional larger population-based studies are needed to confirm these results.

\section{MATERIALS AND METHODS}

\section{Ethics statement}

This study adhered to the principles of the Declaration of Helsinki, and all protocols involving human specimens were approved by the Ethical Committee of the Zhengzhou Traditional Chinese Medicine Traumatology Hospital. Informed consent was obtained from each subject.

\section{Study participants}

285 steroid-induced ONFH patients were enrolled between September 2014 and January 2016 at the Zhengzhou Traditional Chinese Medicine Traumatology Hospital. Steroid-induced ONFH was defined by a history of a mean daily steroid dose of $\geq 16.6 \mathrm{mg}$, or a high-dose steroid impulsion therapy lasting more than 1 week [34-36]. ONFH was diagnosed by examining osteonecrosis in anteroposterior and frog view X-rays of both hips and/or magnetic resonance imaging [37]. Exclusion criteria were as follows: (i) those who drank the equivalent of more than $400 \mathrm{~mL}$ of pure ethanol per week; (ii) those did not meet the diagnostic criteria for steroid-induced ONFH and patients with traumatic ONFH, dislocation of the hip joint, and other hip diseases. (iii) those who had significant familial hereditary diseases.

None of the 507 control subjects recruited based on medical examinations at the Zhengzhou Traditional Chinese Medicine Traumatology Hospital were related to the case subjects. All subjects were Han Chinese from northern China. Subjects with excessive consumption of alcohol, corticosteroids, or significant familial hereditary diseases were excluded.

\section{SNP genotyping}

All eight SNPs had minor allele frequencies $>5 \%$ in the HapMap Chinese Han Beijing (CHB) population. Blood samples were collected in EDTA tubes and stored at $-80^{\circ} \mathrm{C}$ after centrifugation at $2000 \mathrm{rpm}$ for 10 minutes. Genomic DNA was extracted from whole blood using an extraction kit (GoldMag, China) and stored at $-20^{\circ} \mathrm{C}$. DNA quantity was evaluated by spectrophotometry (DU530UV/ VIS spectrophotometer, Beckman Instruments, Fullerton, CA, USA). The Multiplexed SNP Mass EXTEND assay was developed using Sequenom MassARRAY Assay Design 3.0 Software. The primers for the eight selected SNPs are shown in Table 5. A Sequenom MassARRAY RS1000 was used for SNP genotyping according to the manufacturer's protocol.

\section{Statistical analyses}

All statistical analyses were performed using SPSS 19.0 software for Windows (SPSS, Chicago, IL). Allele 
Table 5: Primers used for this study

\begin{tabular}{|c|c|c|c|}
\hline SNP_ID & 1st-PCRP & 2nd-PCRP & UEP_SEQ \\
\hline rs 1053605 & $\begin{array}{c}\text { ACGTTGGATGCTCAAA } \\
\text { GTTGTAGGTGGTGG }\end{array}$ & $\begin{array}{c}\text { ACGTTGGATGAAGGAGTA } \\
\text { CAACAGCTGCAC }\end{array}$ & AACAGCTGCACTGATAC \\
\hline rs243849 & $\begin{array}{l}\text { ACGTTGGATGTACCTTG } \\
\text { GTCAGGGCAGAAG }\end{array}$ & $\begin{array}{c}\text { ACGTTGGATGAGTGACGG } \\
\text { AAAGATGTGGTG }\end{array}$ & ACAGCCAACTACGATGA \\
\hline rs243847 & $\begin{array}{c}\text { ACGTTGGATGAAGACAA } \\
\text { GAGCAGTGACCCC }\end{array}$ & $\begin{array}{c}\text { ACGTTGGATGCCAAAATC } \\
\text { AGACCCTGGTAG }\end{array}$ & сcTGCTGCTACTCACCTCC \\
\hline rs 243832 & $\begin{array}{c}\text { ACGTTGGATGCCTATGCC } \\
\text { AGGCAGAAATTC }\end{array}$ & $\begin{array}{c}\text { ACGTTGGATGGAGAAAGA } \\
\text { AGAGACCGTGAC }\end{array}$ & ACATTCTGGCACACAGAAG \\
\hline rs7201 & $\begin{array}{c}\text { ACGTTGGATGTCCAATCC } \\
\text { CACCAACCCTCA }\end{array}$ & $\begin{array}{c}\text { ACGTTGGATGGCAGGGCTG } \\
\text { CGTTGAAAATA }\end{array}$ & aAGGGCTGCGTTGAAAATATCAAAG \\
\hline rs3918249 & $\begin{array}{c}\text { ACGTTGGATGAAGCACT } \\
\text { GGTGTCTGGAAAG }\end{array}$ & $\begin{array}{c}\text { ACGTTGGATGGATTACAAG } \\
\text { TGTGAGCCGTC }\end{array}$ & gaaGTCATGCCCAGCAGGGACTA \\
\hline rs2274755 & $\begin{array}{c}\text { ACGTTGGATGGGGAGAG } \\
\text { AATGAAGGGAATC }\end{array}$ & $\begin{array}{c}\text { ACGTTGGATGTTCGACGAT } \\
\text { GACGAGTTGTG }\end{array}$ & gCTGGGCAAGGGCGTCGGT \\
\hline rs3918254 & $\begin{array}{c}\text { ACGTTGGATGTCTTCGG } \\
\text { CTTCTGCCCGAC }\end{array}$ & $\begin{array}{c}\text { ACGTTGGATGCAATACATG } \\
\text { ATGAGAGGGCG }\end{array}$ & CTGGTAGACAGGGTGGA \\
\hline
\end{tabular}

and genotype frequencies were determined using direct counts. Hardy-Weinberg equilibrium for each SNP was determined using an exact test to compare the expected frequencies of genotypes in controls. All $p$ values were two-sided, and $p \leq 0.05$ was considered statistically significant. Allele and genotype frequencies in ONFH patients and controls were calculated using Chi-squared test/Fisher's exact tests. Associations between SNPs and risk of steroid-induced ONFH were tested in genetic models using SNP Stats software. Odds ratios (ORs) and 95\% confidence intervals (CIs) were calculated using unconditional logistic regression analysis with adjustment for gender and age. Finally, the Haploview software package (version 4.2) and SHEsis software platform (http:// www.nhgg.org/analysis/) were used to estimate pairwise linkage disequilibrium (LD), haplotype construction, and genetic association at polymorphism loci.

\section{ACKNOWLEDGMENTS AND FUNDING}

This work was supported by the National Natural Science Foundation of China (No. 81160228, 81260284, 81660378). We wish to express heartfelt thanks to all of the patients and healthy control subjects, the clinicians, and the other hospital staff for their participation in and contributions to this study.

\section{CONFLICTS OF INTEREST}

The authors declare no conflicts of interest.

\section{REFERENCES}

1. Cui L, Zhuang Q, Lin J, Jin J, Zhang K, Cao L, Lin J, Yan S, Guo W, He W, Pei F, Zhou Y, Weng X. Multicentric epidemiologic study on six thousand three hundred and ninety five cases of femoral head osteonecrosis in China. Int Orthop. 2016; 40:267-276.

2. Zhao DW, Yu M, Hu K, Wang W, Yang L, Wang BJ, Gao XH, Guo YM, Xu YQ, Wei YS, Tian SM, Yang F, Wang N, et al. Prevalence of Nontraumatic Osteonecrosis of the Femoral Head and its Associated Risk Factors in the Chinese Population: Results from a Nationally Representative Survey. Chinese Med J-Peking. 2015; 128:2843-2850.

3. Garvican ER, Vaughan-Thomas A, Redmond C, Gabriel N, Clegg PD. MMP-mediated collagen breakdown induced by activated protein $\mathrm{C}$ in equine cartilage is reduced by corticosteroids. J Orthop Res. 2010; 28:370-378.

4. Chuang TD, Pearce WJ, Khorram O. miR-29c induction contributes to downregulation of vascular extracellular matrix proteins by glucocorticoids. Am J Physiol-Cell Ph. 2015; 309:C117-125.

5. Liu X, Han Q, Sun R, Li Z. Dexamethasone regulation of matrix metalloproteinase expression in experimental pneumococcal meningitis. Brain Res. 2008; 1207:237-243.

6. He DK, Shen J, Zhang L, Huang WB. Effects of dexamethasone pretreatment on expression of matrix metalloproteinase-9 in rats with acute lung injury induced by phosgene. [Article in Chinese]. Chinese journal of industrial hygiene and occupational diseases. 2011; 29:289-293.

7. Murphy G, Knauper V, Atkinson S, Butler G, English W, Hutton M, Stracke J, Clark I. Matrix metalloproteinases in arthritic disease. Arthritis Res. 2002; 4:S39-49.

8. Grassel S, Beckmann J, Rath B, Vogel M, Grifka J, Tingart M. Expression profile of matrix metalloproteinase-2 and -9 and their endogenous tissue inhibitors in osteonecrotic femoral heads. Int J Mol Med. 2010; 26:127-133.

9. Duerr S, Stremme S, Soeder S, Bau B, Aigner T. MMP2 /gelatinase $A$ is a gene product of human adult articular chondrocytes and is increased in osteoarthritic cartilage. Clin Exp Rheumatol. 2004; 22:603-608. 
10. Vu TH, Shipley JM, Bergers G, Berger JE, Helms JA, Hanahan D, Shapiro SD, Senior RM, Werb Z. MMP-9/ gelatinase $\mathrm{B}$ is a key regulator of growth plate angiogenesis and apoptosis of hypertrophic chondrocytes. Cell. 1998; 93:411-422.

11. Itoh $\mathrm{T}$, Matsuda $\mathrm{H}$, Tanioka $\mathrm{M}$, Kuwabara $\mathrm{K}$, Itohara $\mathrm{S}$, Suzuki R. The role of matrix metalloproteinase- 2 and matrix metalloproteinase-9 in antibody-induced arthritis. J Immunol (Baltimore, Md : 1950). 2002; 169:2643-2647.

12. Yoshihara Y, Nakamura H, Obata K, Yamada H, Hayakawa T, Fujikawa K, Okada Y. Matrix metalloproteinases and tissue inhibitors of metalloproteinases in synovial fluids from patients with rheumatoid arthritis or osteoarthritis. Ann Rheum Dis. 2000; 59:455-461.

13. Al Aqeel A, Al Sewairi W, Edress B, Gorlin RJ, Desnick RJ, Martignetti JA. Inherited multicentric osteolysis with arthritis: a variant resembling Torg syndrome in a Saudi family. Am J Med Genet. 2000; 93:11-18.

14. Zaidi M, Sun L, Robinson LJ, Tourkova IL, Liu L, Wang Y, Zhu LL, Liu X, Li J, Peng Y, Yang G, Shi X, Levine A, et al. ACTH protects against glucocorticoid-induced osteonecrosis of bone. Proc Natl Acad Sci U S A. 2010; 107:8782-8787.

15. Zhou X, Yishake M, Li J, Jiang L, Wu L, Liu R, Xu N. Genetic susceptibility to prosthetic joint infection following total joint arthroplasty: A systematic review. Gene. 2015; 563:76-82.

16. Manso H, Krug T, Sobral J, Albergaria I, Gaspar G, Ferro JM, Oliveira SA, Vicente AM. Variants of the Matrix Metalloproteinase-2 but not the Matrix Metalloproteinase-9 genes significantly influence functional outcome after stroke. BMC Med Genet. 2010; 11:40.

17. Han DH, Kim SK, Kang S, Choe BK, Kim KS, Chung JH. Matrix Metallopeptidase 2 Gene Polymorphism is Associated with Obesity in Korean Population. Korean J Physiol Pha. 2008; 12:125-129.

18. Liu O, Li J, Xin Y, Qin Y, Li H, Gong M, Liu Y, Wang X, Li J, Zhang H. Association of MMP-2 gene haplotypes with thoracic aortic dissection in chinese han population. BMC Cardiovasc Disor. 2016; 16:11.

19. Tsai EM, Wang YS, Lin CS, Lin WY, Hsi E, Wu MT, Juo SH. A microRNA-520 mirSNP at the MMP2 gene influences susceptibility to endometriosis in Chinese women. J Hum Genet. 2013; 58:202-209.

20. Lazary A, Kosa JP, Tobias B, Lazary J, Balla B, Bacsi K, Takacs I, Nagy Z, Mezo T, Speer G, Lakatos P. Single nucleotide polymorphisms in new candidate genes are associated with bone mineral density and fracture risk. Eur J Endocrinol. 2008; 159:187-196.

21. Low SK, Zembutsu H, Takahashi A, Kamatani N, Cha PC, Hosono N, Kubo M, Matsuda K, Nakamura Y. Impact of LIMK1, MMP2 and TNF-alpha variations for intracranial aneurysm in Japanese population. J Hum Genet. 2011; $56: 211-216$.
22. Zhang Y, Wang M, Zhang S. Association of MMP-9 Gene Polymorphisms with Glaucoma: A Meta-Analysis. Ophthalmic Res. 2016; 55:172-179.

23. Jimenez-Morales S, Martinez-Aguilar N, GamboaBecerra R, Jimenez-Ruiz JL, Lopez-Ley D, Lou H, Saldana-Alvarez Y, Dean M, Orozco L. Polymorphisms in metalloproteinase-9 are associated with the risk for asthma in Mexican pediatric patients. Hum Immunol. 2013; 74:998-1002.

24. Gao XJ, Hou SP, Li PH. The association between matrix metalloprotease-9 gene polymorphisms and primary angle-closure glaucoma in a Chinese Han population. Int $\mathrm{J}$ Ophthalmol-Chi. 2014; 7:397-402.

25. Kusano K, Miyaura C, Inada M, Tamura T, Ito A, Nagase H, Kamoi K, Suda T. Regulation of matrix metalloproteinases (MMP-2, -3, -9, and -13) by interleukin-1 and interleukin-6 in mouse calvaria: association of MMP induction with bone resorption. Endocrinology. 1998; 139:1338-1345.

26. Lorenzo JA, Pilbeam CC, Kalinowski JF, Hibbs MS. Production of both $92-$ and $72-\mathrm{kDa}$ gelatinases by bone cells. Matrix (Stuttgart, Germany). 1992; 12:282-290.

27. Wittrant Y, Theoleyre S, Couillaud S, Dunstan C, Heymann D, Redini F. Regulation of osteoclast protease expression by RANKL. Biochem Bioph Res Co. 2003; 310:774-778.

28. Fujisaki K, Tanabe N, Suzuki N, Kawato T, Takeichi O, Tsuzukibashi O, Makimura M, Ito K, Maeno M. Receptor activator of NF-kappaB ligand induces the expression of carbonic anhydrase II, cathepsin K, and matrix metalloproteinase-9 in osteoclast precursor RAW264.7 cells. Life Sci. 2007; 80:1311-1318.

29. Guo LJ, Xie H, Zhou HD, Luo XH, Peng YQ, Liao EY. Stimulation of RANKL and inhibition of membrane-type matrix metalloproteinase-1 expression by parathyroid hormone in normal human osteoblasts. Endocr Res. 2004; 30:369-377.

30. Amalinei C, Caruntu ID, Giusca SE, Balan RA. Matrix metalloproteinases involvement in pathologic conditions. Rom J Morphol Embryo. 2010; 51:215-228.

31. Beeh KM, Beier J, Kornmann O, Buhl R. Sputum matrix metalloproteinase-9, tissue inhibitor of metalloprotinease-1, and their molar ratio in patients with chronic obstructive pulmonary disease, idiopathic pulmonary fibrosis and healthy subjects. Resp Med. 2003; 97:634-639.

32. Zeng R, Zhang X, Wu K, Su Y, Wen F. MMP9 gene polymorphism is not associated with polypoidal choroidal vasculopathy and neovascular age-related macular degeneration in a Chinese Han population. Ophthalmic Genet. 2014; 35:235-240.

33. Nakashima K, Hirota T, Obara K, Shimizu M, Doi S, Fujita K, Shirakawa T, Enomoto T, Yoshihara S, Ebisawa M, Matsumoto K, Saito H, Suzuki Y, et al. A functional polymorphism in MMP-9 is associated with childhood atopic asthma. Biochem Bioph Res Co. 2006; 344:300-307. 
34. Koo KH, Kim R, Kim YS, Ahn IO, Cho SH, Song HR, Park YS, Kim H, Wang GJ. Risk period for developing osteonecrosis of the femoral head in patients on steroid treatment. Clin Rheumatol. 2002; 21:299-303.

35. Eichelbaum M, Fromm MF, Schwab M. Clinical aspects of the MDR1 (ABCB1) gene polymorphism. Ther Drug Monit. 2004; 26:180-185.

36. Zhang Y, Kong X, Wang R, Li S, Niu Y, Zhu L, Chen W, Lin N. Genetic association of the P-glycoprotein gene
$\mathrm{ABCB} 1$ polymorphisms with the risk for steroid-induced osteonecrosis of the femoral head in Chinese population. Mol Biol Rep. 2014; 41:3135-3146.

37. Totty WG, Murphy WA, Ganz WI, Kumar B, Daum WJ, Siegel BA. Magnetic resonance imaging of the normal and ischemic femoral head. Am J Roentgenol. 1984; 143:1273-1280. 